\title{
DISPONIBILIDADE DE FÓSFORO PELOS EXTRATORES DE MEHLICH 1 E RESINA EM LATOSSOLO VERMELHO AMARELO, ÁLICO CULTIVADO COM TRES GRAMÍNERAS FORRAGEIRAS ${ }^{1}$
}

\author{
L. de A. CORRÊA \\ UEPAE de São Carlos - EMBRAPA, C.P. 339 - CEP: 13560-970-Săo Carlos,SP. \\ H.P. HAAG (in memoriam) \\ Departamento de Qulmica - ESALQ/USP - C.P. 9 - CEP: 13418-900-Piracicaba,SP
}

\begin{abstract}
RESUMO: E apresentado um estudo comparando os extratores de Mehlich 1 e Resina ma avaliação do $P$ disponivel em Latossolo Vermelho Amarelo, alico cultivado com as gramíneas Brachiaria decumbens Stapf, Brachiaria brizantha (Hochst.) Stapf. cv. Marandu e Panicum maximum Jacq. Foram utilizados dados de dois ensaios de adubação fosfatada, um realizado em vasos em casa de vegetação e o outro no campo. Correlaçes entre o $P$ extraifdo através de cada extrator com a produção relativa de matéria seca, o P extrádo pela planta e o teor de P na planta, permitiram determinar o extrator de $P$ mais eficiente. Os resultados mostraram que os dois extratores foram eficientes e similares na avaliaçāo do $P$ disponível para as trés gramíneas forrageiras, sendo que o método da Resina apresentou maior capacidade de extração do $P$ aplicado.
\end{abstract}

Descritores: extratores de $P$, gramineas forrageiras.

\section{AVAILABILITY OF PHOSPHORUS BY THE MEHLICH 1 AND RRSTN METHODS IN A RED YELLOW LATOSOL CULTTVATED WITA THREE GRASSES}

ABSTRACT: This study was undertaken to compare the Mehlich 1 and Resin methods in the evaluation of available P in a Red Yellow Latossol, cultivated with Brachiaria decumbens Stapf., Brachiarla brizantha (Hochst.) Stapf cv. Marandu and Panicum maximum Jacq. Data of two experiments of $P$ fertilization were utilized, one carried out in the greenhouse and the other in the field. Correlations between the amount of $P$ extracted by each extrator with the relative production of dry matter and of the amonnt of $P$ extracted by the plant and the level of $P$ in the plant, were used to determine the efficiency of the extractors. The results showed that both extractors were efficient and similar for the evaluation of the $\mathbf{P}$ availability of the three grasses, but the resin method had a greater extraction capacity of the $\mathbf{P}$ applied.

Key Words: phosphorus availability, grasses.

\section{INTRODUÇÃO}

Uma adequada avaliação do $P$ disponível no solo deve incluir os fatores Quantidade $(Q)$ ou $P$ lábil, Intensidade (I) ou $P$ da solução, Capacidade ou poder tampão de $P$, bem como a difusão do elemento no solo (GUNARY \& SUTTON, 1967).

Embora a maioria dos métodos utilizados na extração do $P$ dos solos, baseie-se em determinações que refletem, principalmente o fator Quantidade, existem métodos satisfatórios para avaliar a disponibilidade de $\mathrm{P}$ em solos, pois esse fator 6 o mais importante a ser considerado na análise de P (RAIJ, 1978 e 1989). Segundo esse autor, existem para uso no Brasil, dois métodos principais, profundamente diferentes, que são o método de Mehlich 1 e o da Resina trocadora de ânions.

O extrator de Mehlich 1 ou duplo ácido ou Carolina do Norte, segundo VOLKWEISS \& RAIJ (1977), baseia-se no princípio da dissolução de minerais contendo $\mathrm{P}$ e/ou deslocamento de $\mathrm{P}$ retido nas superfícies sólidas do solo para a solução, por ânions capazes de competir com o P pelos sítios de retenção.

\footnotetext{
${ }^{1}$ Parte da Tese apresentada pelo $1^{\circ}$ Autor para obtenção do título de Doutor em Agronomia - ESALQ/USP - Piracicaba,SP.
} 
Para LOPES et al. (1982), o extrator de Mehlich 1 vem se apresentando razoavelmente adequado como indicador da disponibilidade de $P$ em solos sem adubação e com aplicação de adubos fosfatados solúveis.

Estudo de correlação para métodos de $\mathbf{P}$ disponível para as plantas, em $\mathbf{4 0}$ solos do Estado do Rio Grande do Sul, e os extratores Bray, Olsen, Morgan (modificados) e Mehlich 1, mostrou que os quatro extratores foram eficientes e similares na avaliação da disponibilidade de $P$, sendo o Mehlich 1 o método que melhor estimou o $\mathrm{P}$ nativo (ANGHINONI \& BOHNEN, 1974).

Para RAIJ et al. (1984), o método Mehlich 1 está entre os métodos ácidos de extração de $P$ que apresentam grande vantagem para uso rotineiro, principalmente porque permite obter extratos límpidos que decantam facilmente, dispensando a filtragem das suspensões de solo. Todavia, segundo esses autores, é possível que o uso de extratores ácidos, nas condições brasileiras, torne-se pouco utilizado no futuro, por duas razões principais: 1) com o possível uso crescente de fosfatos naturais, o uso de extratores ácidos como o Mehlich 1, que dissolvem apatita, superestimariam os teores de $\mathbf{P}$ disponível; e 2) em solos adubados é de se esperar que a reserva de $P$ lábil encontre-se em grande parte na forma de fosfatos de Al e Fe, principalmente se os solos forem ácidos, sendo preferível, então,extratores ou métodos que tenham maior ação sobre esta fração, como é o caso dos métodos Bray, da Resina e mesmo do método Olsen.

Para KOCHHANN et al. (1982), a substituição do método de Mehlich 1 por algum outro método testado, resulta numa certa melhoria na avaliação de $P$ disponível, principalmente quando fosfatos pouco solúveis são aplicados. No entanto, a falta de similaridade entre o processo de absorção de nutrientes pelas plantas, com a extração pelos métodos químicos, ainda persiste.

O método da Resina trocadora de ânions foi proposto por AMER et al. (1955), como uma tentativa de reproduzir em laboratório o processo de absorção de $\mathbf{P}$ pelas plantas no campo.

Em uma ampla revisão bibliográfica sobre métodos de extração de P feita por RAlJ (1978), ficou demonstrado que o método mais eficiente para o P, em uma grande variedade de condições, é o baseado na extração da resina trocadora de ânions.

Em estudo comparando os métodos IAC*

-Instituto Agronômico de Campinas, Campinas,SP.
$\left(\mathrm{H}_{2} \mathrm{SO}_{4}, 0,05 \mathrm{~N}\right)$, Bray modificado, Olsen e Resina, através de correlação entre os teores de $P$ nos solos e as respostas à adubação fosfatada, em 32 ensaios de campo, com as culturas de algodão e milho, constatou-se a superioridade do método da Resina (RAIJ et al., 1984).

Um dos incovenientes do método da Resina é o fato de ser um método trabalhoso. Todavia, valem esforços para a sua utilização, pois apresenta melhor correlação com respostas à adubação fosfatada, analogia com a extração da planta muito superior aos outros métodos, além do aspecto positivo de não incluir nenhum agente químico de ação específica sobre os fosfatos do solo (RAIJ et al., 1982 e 1984).

O estudo desenvolvido em vasos em casade-vegetação e a campo teve o objetivo de comparar a eficiência dos extratores de Mehlich 1 e Resina na avaliação do $P$ disponivel para três gramíneas forrageiras em Latossolo Vermelho Amarelo, álico.

\section{MATERIAL E MÉTODOS}

Foram utilizados dados de dois ensaios de adubação fosfatada, um realizado $\mathrm{cm}$ vasos $\mathrm{cm}$ casa-de-vegetação c o outro no campo, em Latossolo Vermelho Amarelo, álico, cultivado com as gramíneas Brachiaria decumbens Stapf., Brachiaria brizantha (Hochst.) Stapf cv. Marandu e Panicum maximum Jacq., cujos resultados c detalhes da metodologia são apresentados por CORRÊA \& HAAG (1993a,b). As características físicas e químicas da amostra de solo coletada na camada de $0-20 \mathrm{~cm}$ de profundidade foram as seguintes: argila $30,7 \%$; silte $13,7 \%$ e areia $55,6 \% ; \mathrm{pH}\left(\mathrm{CaCl}_{2}\right) 4,2, \mathrm{Ca}^{12} \mathbf{0}, 33 ; \mathrm{Mg}^{+2} 0,28 ; \mathrm{K}^{+}$ 0,$12 ; \mathrm{H}^{+} \mathrm{Al} 5,07$ e CTC $3,2 \mathrm{meq} / 100 \mathrm{~cm}^{3}$ e P 2,5 ppm (Resina). No ensaio em vasos foram aplicadas doses de $P$ de $0 ; 17,5 ; 35 ; 70 ; 140 ; 280$; e 560 ppm equivalentes respectivamente a $0,100,200$, $400,800,1600$ e $3200 \mathrm{~kg}$ de $\mathrm{P}_{2} \mathrm{O}_{5} / \mathrm{ha}$. No ensaio a campo foram aplicadas doses de $\mathrm{P}$ correspondente a $0,20,40,80,160,320$ e $640 \mathrm{~kg}$ de $\mathrm{P}_{2} \mathrm{O}_{5} / \mathrm{ha}$. Nos dois ensaios, a fonte de $P$ utilizada foi o superfosfato triplo granulado, sendo aplicado também calcário, nitrogênio, polássio e zinco.

Foram feitas amostragens de solo após o primeiro e o segundo cortes de avaliação da produção de matéria seca das plantas. Cada amostra de solo foi analisada e determinado o teor de $\mathrm{P}$ disponivel pelo extrator de Mehlich $1 \mathrm{(H}_{2} \mathrm{SO}_{4}$ $0.025 \mathrm{~N}+\mathrm{HCl} 0,05 \mathrm{~N}$ ) na proporção de $10 \mathrm{~cm}^{3} \mathrm{de}$ 
terra para $100 \mathrm{ml}$ da solução extratora, segundo o método da EMPRESA BRASILEIRA DE PESQUISA AGROPECUÁRIA (1979), e pelo método da "resina trocadora de ânions", com um tempo de agitação de 16 horas, segundo RAIJ \& QUAGGIO (1983).

Pelo estudo de correlação entre o P extraído através de cada extrator e a produção relativa de matéria seca, $P$ extraído pela planta e teor de P na planta, foi determinado o extrator de $\mathbf{P}$ mais adequado para avaliar o $\mathbf{P}$ disponível para as três espécies forrageiras.

Com o propósito de estabilizar as variâncias entre doses, as variáveis teor de $P$ no solo, teor de $\mathbf{P}$ na planta e $\mathbf{P}$ extraído pela planta foram transformadas através de logarítimo neperiano e quando necessário para interpretação, essas variáveis foram retransformados para a escala original.

\section{RESULTADOS E DISCUSSÃO}

\section{Ensaio em vasos em casa de regetação}

O P extraído pelos métodos de Mehlich 1 e da Resina nas amostras de solo, coletadas após cada corte das plantas, aumentou de forma quadrática $(P<0,01)$ com o aumento das doses de $P$ aplicadas, obtendo-se uma elevada correlação entre o P aplicado e P extraído, para os dois extratores (TABELA 1). Verifica-se que, independentemente da gramínea cultivada, do corte e principalmente nas doses de $\mathbf{P}$ mais elevadas, 0 método da Resina apresentou maior capacidade de extração do $P$ aplicado que o método de Mehlich 1. As diferenças observadas na capacidade de extração do $P$ adicionado, são devidas aos diferentes processos de ação desses métodos. $O$ método Mehlich 1, que $\varepsilon$ um processo químico, atua preferencialmente sobre determinadas formas de $\mathbf{P}$ no solo. O método da Resina, por sua vez, atua pelo sistema de troca iônica, extraindo de modo indiscriminado o $P$ lábil. No presente estudo, o solo utilizado foi um Latossol Vermelho Amarelo, álico, rico em $6 x i d o s$ de ferro e alumínio, adubado com uma fonte solúvel de $P$. Nesta situação é de se esperar que a reserva de $P$ lábil esteja principalmente nas formas de fosfatos de $\mathrm{Fe}$ e $\mathrm{Al}$, que são mais removidas pelo método da Resina, do que pelo Mehlich 1, que tem uma ação preferencial sobre fosfatos de cálcio.

Para avaliar a eficiência dos métodos, foram feitos estudos de correlação entre o $P$ extraído através de cada extrator $\mathrm{e}$ o $\mathrm{P}$ extraído pela planta, porcentagem de $P$ na parte aérea $e$ produção relativa de matéria seca para cada gramínea, nos dois cortes de avaliação. Os coeficientes de correlação encontrados estão na TABELA 2.

Verifica-se pelos elevados coeficientes de correlação e pela semelhança nos valores obtidos, que os dois extratores foram eficientes e similares na avaliação do $P$ disponível para as três gramíneas. Essa similaridade $\epsilon$ confirmada pela elevada correlação obtida entre os extratores, que foi em média, de 0,97. De maneira geral, dentre os parâmetros de plantas estudados, o $\mathrm{P}$ extraído pela planta e a porcentagem de $P$ na parte aérea, foram os mais adequados na avaliação da eficiência dos dois métodos, pois apresentaram um maior coeficiente de correlação com o P extraído pelos dois extratores.

Embora os dois extratores tenham apresentado condições semelhantes de utilização para este solo, o método da Resina pela sua maior capacidade de extração, seria o mais indicado em estudos de calibração, uma vez que segundo KOCHHANN et al. (1982), métodos com essa característica apresentam uma menor possibilidade de ocorrência de erros analíticos, devido às maiores faixas de classes de $P$ obtidas.

Observa-se também, que de maneira geral, ocorreu reduzida extração de $P$, para os dois extratores, nas doses mais baixas de $P$ aplicadas, $a$ qual só aumentou mais acentuadamente a partir da dose de 70 ppm de P (400 kg de $\mathrm{P}_{2} \mathrm{O}_{5} / \mathrm{ha}$ ).

A fim de se estudar a taxa de recuperação do $P$ aplicado, determinou-se a porcentagem de $P$ recuperado através da fórmula:

$$
\% \mathrm{P} \text { recup. }=\frac{\mathrm{P} \text { na anál. }-\mathrm{P} \text { nat. }}{\mathrm{P} \text { aplic. }} \times 100,
$$

cujos resultados estão na TABELA 3. Os cálculos foram feitos com a média dos valores de $P$ extraído nas amostras de solo das três gramíneas, tendo em vista que a tendência de extração do $P$ pelos dois extratores foi a mesma, independentemente da gramínea cultivada.

Confirma-se na TABELA 3, que nas doses mais baixas de $P$ aplicadas, a porcentagem de recuperação é muito baixa, principalmente no caso do extrator de Mehlich 1. Esse fato $E$ uma indicação de que quando se usam doses relativamente baixas de adubo fosfatado, ou solos com teores relativamente baixos de $P$, situação 
TABELA 1 - Equações de regressão ajustadas para o P extraído (y), transformado para ln em função das doses de $\mathbf{P}$ aplicadas (x), após o primeiro e o segundo cortes de cada gramínea, de acordo com o extrator.

\begin{tabular}{|c|c|c|c|}
\hline Extrator & Gramíneas & Equações & $\mathbf{R}^{2}$ \\
\hline \multirow{6}{*}{ Mehlich 1} & B.decumbens ${ }^{1} * *$ & $y=1,065+0,00292000 x-0,00000050 x^{2}$ & 0,97 \\
\hline & B. decumbens ${ }^{2} * *$ & $y=1,163+0,00236801 x-0,00000035 x^{2}$ & 0,98 \\
\hline & B. brizantha ${ }^{1} *$ & $y=1,014+0,00231082 x-0,00000035 x^{2}$ & 0,95 \\
\hline & B.brizantha $a^{2} * *$ & $y=1,251+0,00219240 x-0,00000031 x^{2}$ & 0,99 \\
\hline & P.maximum ${ }^{1} * *$ & $y=0,837+0,00253829 x-0,00000037 x^{2}$ & 0,98 \\
\hline & P.maximum ${ }^{2} * *$ & $y=1,299+0,00222935 x-0,00000032 x^{2}$ & 0,98 \\
\hline \multirow{6}{*}{ Resina } & B.decumbens ${ }^{1} * *$ & $y=1,330+0,00324610 x-0,00000061 x^{2}$ & 0,97 \\
\hline & B. decumbens ${ }^{2} * *$ & $y=1,457+0,00276568 x-0,00000048 x^{2}$ & 0.99 \\
\hline & B.brizantha $a^{1} * *$ & $y=1,430+0,00265441 x-0,00000046 x^{2}$ & 0,88 \\
\hline & B.brizantha ${ }^{2} * *$ & $y=1,548+0,00277827 x-0,00000050 x^{2}$ & 0,99 \\
\hline & P.maximum ${ }^{1} * *$ & $y=1,178+0,00289870 x-0,00000049 x^{2}$ & 0,93 \\
\hline & P.maximum ${ }^{2} * *$ & $y=1,499+0,00286301 x-0,00000053 x^{2}$ & 0,98 \\
\hline
\end{tabular}

$*(\mathrm{P}<0,05) ; * *(\mathrm{P}<0,01) ; 1-1^{\circ}$ Corte $; 2-2^{\circ}$ Corte

TABELA 2 - Coeficientes de correlação ${ }^{1}$ entre o $\mathrm{P}$ extraído pelos métodos Mehlich 1 e Resina e teor de $\mathrm{P}$ na parte aérea (TP), $\mathrm{P}$ extraído pela planta (PE) e produção relativa (PR) de cada gramínea, nos dois cortes de avaliação.

\begin{tabular}{cccccc}
\hline \hline \multirow{2}{*}{ Espécie } & & \multicolumn{5}{c}{ Métodos } \\
\cline { 3 - 6 } & Variável & \multicolumn{3}{c}{ Mehlich 1} & \multicolumn{2}{c}{ Resina } \\
\cline { 3 - 6 } & & $1^{\circ}$ Corte & $2^{\circ}$ Corte & $1^{\circ}$ Corte & $2^{\circ}$ Corte \\
\hline \multirow{2}{*}{ B.decumbens } & TP & 0,98 & 0,99 & 0,94 & 0,97 \\
& PE & 0,95 & 0,96 & 0,95 & 0,96 \\
& PR & 0,69 & 0,70 & 0,69 & 0,70 \\
B.brizantha & TP & 0,97 & 0,92 & 0,98 & 0,97 \\
& PE & 0,91 & 0,90 & 0,95 & 0,96 \\
& PR & 0,71 & 0,71 & 0,70 & 0,72 \\
P.maximum & TP & 0,90 & 0,94 & 0,94 & 0,98 \\
& PE & 0,82 & 0,90 & 0,88 & 0,97 \\
& PR & 0,68 & 0,71 & 0,68 & 0,72 \\
\hline \hline 1-(P<0,01) & & & & &
\end{tabular}


TABELA 3 - Porcentagem média de $P$ recuperado pelos extratores de Mehlich 1 e Resina, em função das doses de $\mathbf{P}$ aplicadas, após o primeiro e segundo cortes das plantas.

\begin{tabular}{|c|c|c|c|c|c|}
\hline \multirow{3}{*}{$\begin{array}{l}\text { Doses de } \mathrm{P} \\
\text { equivalentes a } \\
\mathrm{kg} \mathrm{de} \mathrm{P}_{2} \mathrm{O}_{5} / \mathrm{ha}\end{array}$} & \multirow{3}{*}{$\begin{array}{c}\text { ppm } \\
\mathbf{P}\end{array}$} & \multicolumn{4}{|c|}{ Extratores } \\
\hline & & \multicolumn{2}{|c|}{ Mehlich 1} & \multicolumn{2}{|c|}{ Resina } \\
\hline & & $1^{\circ}$ Corte & $2^{\circ}$ Corte & $1^{\circ}$ Corte & $2^{\circ}$ Corte \\
\hline & & & $\%$ & - & - \\
\hline 0 & 0 & - & - & - & - \\
\hline 100 & 17,5 & 4,8 & 4,8 & 7,0 & 8,2 \\
\hline 200 & 35 & 5,1 & 5,5 & 8,0 & 9,2 \\
\hline 400 & 70 & 6,4 & 6,7 & 10,5 & 11,6 \\
\hline 800 & 140 & 10,0 & 9,7 & 17,3 & 18,6 \\
\hline 1600 & 280 & 21,0 & 19,0 & 37,6 & 37,3 \\
\hline 3200 & 560 & 29,3 & 29,7 & 41,0 & 34,0 \\
\hline
\end{tabular}

comum no caso de pastagens, os extratores, principalmente o Mehlich 1, podem não refletir adequadamente a quantidade de adubo que foi ou deve ser aplicada aos solos.

\section{Ensaio de campo}

O P extraído pelos métodos de Mehlich 1 e Resina, das amostras de solo, coletadas após cada corte das plantas, aumentou de forma linear $e$ quadrática $(P<0,01)$, de acordo com as doses de $P$ aplicadas (TABELA 4). Verifica-se que, da mesma forma que ocorreu no ensaio em vasos, o método da Resina apresentou maior capacidade de extração do $P$ aplicado do que 0 método de Mehlich 1. Essas diferenças se devem aos diferentes processos de extração de $P$ dos dois métodos, conforme já discutido no caso do ensaio em vasos.

Para avaliar a eficiência dos métodos, foram feitos estudos de correlação entre $O P$ extraído por cada extrator e o $P$ extraído pela planta,porcentagem de $P$ na parte aérea e produção relativa de matéria seca de cada gramínea, nos dois cortes de avaliação. Os coeficientes de correlação encontrados estão na TABELA 5.

Verifica-se, pelos coeficientes de correlação e pela semelhança nos valores obtidos, que os dois extratores foram eficientes e similares na avaliação do $P$ disponível para as três gramíneas, nos dois cortes de avaliação.
Essa similaridade entre os extratores é confirmada pelo elevado coeficiente de correlação obtido entre os extratores, que foi em média de 0,98 .

Dentre os parâmetros de planta estudados, o P extraído (PE) e porcentagem de $P$ na parte aérea (TP), foram os mais eficientes, mostrado pelos maiores coeficientes de correlação com o $P$ extraído pelos dois extratores.

Embora os dois extratores tenham apresentado semelhantes condições de utilização para este solo, o método da Resina seria o mais indicado, em estudos de calibração, pela sua maior capacidade de recuperação do $P$, conforme já discutido no texto referente ao ensaio em vasos.

Verifica-se que, de maneira geral, ocorreu também pouca extração do $P$ aplicado nas doses mais baixas, a qual só aumentou mais acentuadamente a partir da dose em torno de 160 $\mathrm{kg} \mathrm{de} \mathrm{P}_{2} \mathrm{O}_{5} / \mathrm{ha}$. Esse fato pode ser melhor avaliado pela porcentagem de $P$ recuperado, cujos cálculos seguiram os mesmos procedimentos feitos para o ensaio em vasos. Nas doses inferiores de $P$ aplicado, a recuperação do $P$ é muito baixa, principalmente no caso do extrator Mehlich 1 (TABELA 6). Desta forma,embora os dois extratores tenham sido eficientes e similares na avaliação do $\mathbf{P}$ disponível para as três gramíneas, no caso da utilização de doses baixas de adubo fosfatado, ou solos com teores relativamente baixos de $P$, situação comum no caso de pastagens, os 
TABELA 4 - Equações de regressão ajustadas para o $P$ extraído (y), transformado para $\ln$, em função das doses de fósforo aplicadas (x), após o primeiro e o segundo cortes de cada gramínea, de acordo com o extrator.

\begin{tabular}{|c|c|c|c|}
\hline Extrator & Gramíneas & Equações ${ }^{3}$ & $\mathbf{R}^{2}$ \\
\hline \multirow{6}{*}{ Mehlich 1} & B.decumbens ${ }^{1}$ & $y=1,118896+0,003999 x$ & 0,90 \\
\hline & B.decumbens ${ }^{2}$ & $y=0,953626+0,004331 x$ & 0,98 \\
\hline & B.brizantha ${ }^{1}$ & $y=1,029576+0,004136 x$ & 0,97 \\
\hline & B.brizantha ${ }^{2}$ & $y=1,025149+0,004124 x$ & 0,96 \\
\hline & P.maximum ${ }^{1}$ & $y=1,099484+0,004145 x$ & 0,98 \\
\hline & P.maximum ${ }^{2}$ & $y=1,071171+0,004367 x$ & 0,97 \\
\hline \multirow{6}{*}{ Resina } & B.decumbens ${ }^{1}$ & $y=1,518805+0,008653 x-0,0000065 x^{2}$ & 0,96 \\
\hline & B.decumbens $s^{2}$ & $y=1,66370+0,0041738 x$ & 0,98 \\
\hline & B.brizantha ${ }^{1}$ & $y=1,546586+0,004712 x$ & 0,96 \\
\hline & B.brizantha ${ }^{2}$ & $y=1,600117+0,004023 x$ & 0,98 \\
\hline & P.maximum ${ }^{1}$ & $y=1,766808+0,004397 x$ & 0,96 \\
\hline & P.maximum ${ }^{2}$ & $y=1,515540+0,00820 x-0,0000059 x^{2}$ & 0,97 \\
\hline
\end{tabular}

$1-1^{0}$ corte $; 2-2^{\circ}$ corte $; 3-(\mathrm{P}<0,01)$.

TABELA 5 - Coeficientes de correlação' entre o P extraído pelos métodos Mehlich I e Resina e teor de $\mathrm{P}$ na parte aérea (TP), $\mathrm{P}$ extraído pela planta (PE) e produção relativa (PR) de cada gramínea, nos dois cortes de avaliação.

\begin{tabular}{cccccc}
\hline \hline \multirow{2}{*}{ Espécie } & \multirow{2}{*}{ Variável } & \multicolumn{3}{c}{ Mehlich 1 } & \multicolumn{2}{c}{ Resina } \\
\cline { 3 - 6 } & & $1^{\circ}$ Corte & $2^{\circ}$ Corte & $1^{\circ}$ Corte & $2^{\circ}$ Corte \\
\hline \multirow{2}{*}{ B.decumbens } & TP & 0,82 & 0,96 & 0,96 & 0,96 \\
& PE & 0,80 & 0,90 & 0,91 & 0,90 \\
& PR & 0,72 & 0,73 & 0,76 & 0,73 \\
B.brizantha & TP & 0,97 & - & 0,97 & - \\
& PE & 0,96 & - & 0,95 & - \\
& PR & 0,81 & - & 0,80 & - \\
& TP & 0,96 & 0,97 & 0,96 & 0,99 \\
& PE & 0,96 & 0,90 & 0,96 & 0,96 \\
& PR & 0,86 & 0,74 & 0,86 & 0,75 \\
\hline \hline
\end{tabular}

$1-(P<0,01)$ 
TABELA 6 - Porcentagem média de P recuperado pelos extratores Mehlich 1 e Resina, em função das doses de $\mathbf{P}$ aplicadas, após o primeiro e segundo cortes das plantas.

\begin{tabular}{|c|c|c|c|c|}
\hline \multirow{2}{*}{$\begin{array}{c}\mathrm{P} \text { aplicado } \\
\mathrm{kg} \text { de } \mathrm{P}_{2} \mathrm{O}_{5} / \mathrm{ha}\end{array}$} & \multicolumn{2}{|c|}{ Mehlich 1} & \multicolumn{2}{|c|}{ Resina } \\
\hline & $1^{\circ}$ Corte & $2^{\circ}$ Corte & $1^{\circ}$ Corte & $2^{\circ}$ Corte \\
\hline 0 & - & - & - & - \\
\hline 20 & 8,5 & 8,6 & 17,0 & 17,1 \\
\hline 40 & 8,6 & 8,6 & 18,6 & 17,1 \\
\hline 80 & 8,6 & 8,6 & 21,0 & 18,6 \\
\hline 160 & 10,4 & 10,0 & 26,0 & 23,0 \\
\hline 320 & 15,0 & 14,6 & 40,0 & 35,0 \\
\hline 640 & 36,1 & 36,0 & 76,7 & 61,0 \\
\hline
\end{tabular}

extratores, principalmente o Mehlich 1, podem não refletir adequadamente a quantidade de adubo que foi ou deve ser adicionado ao solo.

No caso das doses de $P$ mais baixas, de $0,20,40$ e $80 \mathrm{~kg}$ de $\mathrm{P}_{2} \mathrm{O}_{5} / \mathrm{ha}$, a quantidade de $\mathrm{P}$ indicado pelos extratores de Mehlich 1 e Resina, no primeiro corte, foi respectivamente de 2,$9 ; 3,2$; 3,5 e 4,1 ppm e de 5,0;5,6; 6,3 e 7,9 ppm, onde se confirma a pouca habilidade, principalmente do extrator de Mehlich 1, para indicar o P adicionado.

SegundoSANCHEZ\& SALINAS (1981), nesta situação há dificuldades em se fazer a recomendação da adubação fosfatada baseada apenas no resultado de análise de solo.

Pelo observado no presente trabalho, quando um resultado de análise de solo, nas condições estudadas, indicar valores relativamente baixos de $P$ disponível, principalmente pelo extrator de Mehlich 1, julga-se que outras informações, como o histórico da área, são necessárias para que se possa fazer uma recomendação mais segura da adubação fosfatada.

\section{CONCLUSÕES}

Os extratores Mehlich 1 e Resina foram eficientes e similares na avaliação do $P$ disponível para as três gramíneas forrageiras, sendo que o método da Resina apresentou maior capacidade de extração do $\mathrm{P}$ aplicado.

\section{REFERÊNCIAS BIBLIOGRÁFICAS}

AMER, F.; BOULDIN, C.A.; BLACK, C.A.Q.; DUCKE, F.R. Characterization of soil phosphorus by anion exchange resin absortion and $\mathbf{P}^{32}$ equilibration. Plant and Soil, Dordrecht, v.6, p.391-407, 1955.

ANGHINONI, I.; BOHNEN, H. Avaliação da disponibilidade do fósforo para os solos do Rio Grande do Sul. Agronomia Sulriograndense, Porto Alegre, v.10, n.1, p.127-336, 1974.

CORREA, L. de A.; HAAG, H.P. Niveis críticos de fósforo para estabelecimentode Brachiaria decumbens Stapf., Brachiaria brizantha (Hochst.) Stapf. cv. Marandu e Panicum maximum Jacq., em Latossolo Vermelho Amarelo, Álico. I. Ensaio em casa de vegetação. Scientia Agricola, Piracicaba. v.50, n.1, p.99-108, 1993a.

CORREA, L. de A.; HAAG, H.P. Níveis críticos de fósforo para estabelecimento de Brachiaria decumbens Stapf., Brachiaria brizantha (Hochst.) Stapf. cv. Marandu e Panicum maximum Jacq., em Latossolo Vermelho Amarelo, Álico. I. Experimento de campo. Scientia Agricola, Piracicaba. v.50, n.1, p.109-116, $1993 b$.

EMPRESA BRASILEIRA DE PESQUISA AGROPECUÁRIA. Serviço Nacional de Levantamento e Conservação de Solos. Manual de métodos de andilises de solo. Rio de Janeiro, 1979. n.p. 
GUNARY, D.; SUTTON, G.D. Soil factors affecting plant uptake of phosphate. Journal of Soil Science, Oxford, v.18, p.167-173, 1967.

KOCHHANN, R.: ANGHINONI,I.; MIELNICZVK, J. Adubação fosfatada no Rio Grande do Sul e Santa Catarina. In: OLIVEIRA, A.J.; LOURENÇO, S.; GOEDERT, W.J., ed. Adubaçăo fosfatada no Brasil. Brasilia, EMBRAPA-DID, 1982. p.29-60, (EMBRAPA-DID. Documentos, 21).

LOPES, A.S.; VASCONCELLOS, C.A.; NOVAIS, R.F. Adubação fosfatada em algumas culturas nos Estados de Minas Gerais, Espirito Santo e Rio Janeiro. In: OLIVEIRA, A.J. de; LOURENÇO, S.; GOEDERT, W.J. Adubação fosfatada no Brasil. Brasilia, EMBRAPA-DID, 1982. p.137-200, (EMBRAPADID. Documentos, 21)

RAIJ, B. van. Seleção de métodos de laboratório para avaliar a disponibilidade de fósforo em solos. Revista Brasileira de Ciencia do Solo, Campinas, v.2, p.1-9, 1978.

RAI, B. van. Fósforo do solo. In: BULL, L.T.; ROSALEM, C.A. Interpretação de andlise química de solos e planta para fins de adubação. Botucatu, Fundação de Estudos e Pesquisas Agrícolas e Florestais, 1989. p.75-96.
RAJ, B. van; QUAGGIO, J.A. Metodo de análise de solo para fins de fertilidade. Campinas, Instituto Agronômico, 1983. 31p. (IAC. Boletim, 81).

RAU, B. van; FEITOSA, C.T.; CARMELLO, Q.A.C. $A$ adubação fosfatada no Estado de São Paulo. In: OLIVEIRA, A.J. de; LOURENÇO, S.; GOEDERT, W.J. (ed.). Adubação fosfatada no Brasil. Brasília, 1982. p.103-36, (EMBRAPA-DID. Documentos, 21).

RAU, B. van; FEITOSA, C.T.; SILVA, N.M. da. Comparação de quatro extratores de fósforo de solos. Bragantia, Campinas, v.43, n.1, p.17-29, 1984.

SANCHEZ, P.A.; SALINAS, J.G. Low-input technology for managing oxisols and ultisols in Tropical America. Advances in Agronomy, San Diego, v.34, p.279-406, 1981.

VOLKWEISS, S.J.; RAIJ, B. van. Retenção e disponibilidade de fósforo em solos. In: SIMPÓSIO SOBRE O CERRADO: BASES PARA A UTILIZAÇĀOAGROPECUARIA, 4., 1976, Brasilia, São Paulo, EDUSP; Belo Horizonte, Ed. Itatiaia, 1977. p.317-32.

Trabalho enviado para publicação em 10.08.92

Trabalho aceito para publicação em 04.12.92 